\title{
On the Order of Singular Optimal Control Problems ${ }^{1}$
}

\author{
W. F. POWERS ${ }^{2}$ \\ Dedicated to R. Bellman
}

\begin{abstract}
In singular optimal control problems, the functional form of the optimal control function is usually determined by solving the algebraic equation which results by successively differentiating the switching function until the control appears explicitly. This process defines the order of the singular problem. Order-related results are developed for singular linear-quadratic problems and for a bilinear example which gives new insights into the relationship between singular problem order and singular arc order.
\end{abstract}

Key Words. Optimal control, singular control, junction conditions, singular control order.

\section{Introduction}

The concept of order for singular problems was introduced in the midsixties when an extension of the generalized Legendre-Clebsch condition (GLC), developed by Kelley in 1964 in Ref. 1, was developed by Kopp and Moyer in Ref. 2. Problem-oriented definitions of order were then developed in Refs. 3 and 4. In 1971, a junction theorem for singular and nonsingular arcs was presented (Ref. 5) in terms of the genericity (i.e., even or odd) of singular problem order and continuity properties of the optimal control function. It should be noted that all of the authors of Refs. 1-5 were concerned with solving singular aerospace optimal control problems at the time of the theoretical developments, and the results of Refs. 1-5 were all applied to realistic engineering problems.

In recent years, studies of order associated with singular problems per se (Refs. 6 and 7) have been presented to develop a deeper understanding of

\footnotetext{
${ }^{1}$ This work was supported by the National Science Foundation under Grant No. ENG-7716660.

${ }^{2}$ Professor, Department of Aerospace Engineering, University of Michigan, Amn Arbor, Michigan, and Manager, Control Systems Department, Research, Ford Motor Company, Dearborn, Michigan.
} 
optimal control theory. In this same spirit, Lewis in Ref. 8 surveys the various definitions and implementations of singular control order. As shown in Ref. 8, basically two types of order are involved in Refs. 1-5: problem order and arc order (to be defined in Section 2). Lewis then presents a bilinear control example for which he claims the junction theorem of Ref. 5 does not apply; and, based upon this fact, one should refrain (at least at the present time) from any claims involving genericity. However, a more complete analysis of this example, to be presented in Section 3, shows that the junction theorem of Ref. 5 is applicable to almost all nonsingularsingular junctions, and that genericity gives a great deal of engineering insight into the problem. Thus, Ref. 8 does not refute order and genericity arguments, which practitioners of optimal control have employed for insights into engineering problems over the last decade.

Additional insights into the order of singular problems are presented in Section 4. In particular, results for a class of infinite-order singular problems are discussed.

\section{Definitions of Order}

Consider the following Bolza problem: minimize

$$
J=G\left(t_{f}, x_{f}\right)+\int_{t_{0}}^{t_{f}}\left[L_{0}(t, x)+L_{u}(t, x) u\right] d t,
$$

subject to

$$
\begin{gathered}
\dot{x}=f_{0}(t, x)+f_{u}(t, x) u, \\
|u(t)| \leq K(t), \quad t \in\left[t_{0}, t_{f}\right], \\
\left\{t_{0}, x\left(t_{0}\right), t_{f}, x\left(t_{f}\right)\right\} \in S,
\end{gathered}
$$

where $x$ is an $n$-vector, $u$ is a scalar control variable, and $K(t)$ is a piecewise analytic function. The Hamiltonian for this problem is

$$
H=L_{0}(t, x)+L_{u}(t, x) u+\lambda^{T}\left[f_{0}(t, x)+f_{u}(t, x) u\right],
$$

and an optimal subarc on which the switching function vanishes,

$$
\begin{aligned}
\phi(t) & \equiv L_{u}\left(t, x^{*}(t)\right)+\lambda^{T}(t) f_{u}\left(t, x^{*}(t)\right)=H_{u}\left[t, x^{*}(t), \lambda(t)\right] \equiv 0, \\
t & \in\left[t_{1}, t_{2}\right], \quad t_{1} \neq t_{2},
\end{aligned}
$$

is called an optimal singular subarc; here, $x^{*}(t)$ and $u^{*}(t)$ denote the optimal state and control, respectively. In the previous section, a historical development of singular problem order was presented. The accepted 
engineering concept of singular order is made precise in the following definition.

Definition 2.1. Assume that the problem defined by Eqs. (1)-(4) contains at least one optimal singular subarc. Let $\left(d^{2 q} / d t^{2 q}\right)\left[H_{u}(t, x, \lambda)\right]$ be the lowest-order total derivative of $H_{b}$ in which $u$ appears explicitly. Then, the optimal control problem is said to be a singular problem of order $q$. If $u$ never appears explicitly in the differentiation process, then the optimal control problem is called an infinite-order singular problem.

Comment 2.1. As is well known, it is necessary that the first appearance of $u$ in the switching function differentiation process must occur in an even-order derivative (see Ref. 4). It is implicit in the definition above that, if $q<\infty$, then $\left(d^{2 q} / d t^{2 q}\right)\left[H_{u}(t, x, \lambda)\right]$ is linear in $u$; in particular,

$$
\left(d^{2 q} / d t^{2 q}\right)\left[H_{u}(t, x, \lambda)\right] \equiv A(t, x, \lambda)+B(t, x, \lambda) u .
$$

Comment 2.2. This definition refines the definitions of Refs. 5 and 9 to emphasize that the accepted concept of order is really associated with the problem, as opposed to an optimal subarc, and thus may be computed a priori. This distinction will become more important in Section 3. Lewis refers to this order as intrinsic order.

Suppose that a singular problem is of $q$ th order, with $q<\infty$. As will be shown in the next section, such a probiem may possess optimal singular subarcs with orders equal to or different from the problem order. Thus, the following definition is proposed for the order of a singular arc in Ref. 8.

Definition 2.2. Let $u^{*}(t), t \in\left[t_{1}, t_{2}\right]$, be an optimal singular control for the $q$ th order singular problem defined by Eqs. (1)-(4). If $q=\infty$, then the subarc is called an infinite-order, singular subarc. If $q<\infty$ and $B\left[t, x^{*}(t), \lambda^{*}(t)\right] \neq 0$, on $\left(t_{1}, t_{2}\right)$, then the subarc is called a $q$ th order singular subarc. If $q<\infty$ and $B\left[t, x^{*}(t), \lambda^{*}(t)\right] \equiv 0, t \in\left(t_{1}, t_{2}\right)$, then the subarc is called a $p$ th order singular subarc, where $p$ is determined as follows. Differentiate $H_{u}^{(2 q)}\left[t, x^{*}(t), \lambda^{*}(t), u^{*}(t)\right]$ with respect to $t$ until

$B_{1}(t) \equiv\left[(\partial / \partial u)\left[H_{u}^{\left(2 q+2 p_{1}\right)}(t, x, \lambda, u)\right]_{\left(t, x^{*}(t), \lambda^{*}(t), u^{*}(t)\right)} \neq 0, \quad t \in\left(t_{1}, t_{2}\right)\right.$.

Then, (i) if $p_{1}<\infty, p=q+p_{1}$; and (ii) if no finite value of $p_{1}$ satisfies Eq. (8), $p=\infty$.

Comment 2.3. In general, the order of a singular arc cannot be determined a priori. However, the arc order is the order of interest in the 
development of necessary conditions which involve expansions about reference optimal solutions.

Comment 2.4. In general, $H_{u}^{\left(2 q+2 p_{1}\right)}[t, x, \lambda, u]$ is a $\left(2 p_{1}+1\right)$-polynomial in $u$, while $H_{u}^{(2 q)}$ is linear in $u$.

With the above definitions, the major theorem of Ref. 5 may be restated as follows.

Theorem 2.1. Let $t_{c}$ be a point at which singular and nonsingular subarcs of an optimal control $u^{*}(t)$ are joined. If the order of the singular problem and the singular subarc are equal, say $q$, if $B\left[t_{c}, x^{*}\left(t_{c}\right), \lambda^{*}\left(t_{c}\right)\right] \neq 0$, and if the control is piecewise analytic in a neighborhood of $t_{c}$, then $q+r$ is an odd integer, where $\left(d^{r} / d t^{r}\right) u^{*}(t), r \geq 0$, is the lowest-order time derivative of $u^{*}(t)$ which is discontinuous at $t_{c}$.

At the present time, there does not exist a general junction necessary condition for singular problems in which the problem and arc orders differ. However, the insights to be discussed in Sections 3 and 4 may allow the generation of such a result in the near future.

\section{Finite-Order Considerations}

As noted previously, a survey of the various definitions and implementations of singular order is presented in Ref. 8. Lewis presents an example, to be discussed below, which he claims represents a class of problems whose junction point behavior is not specified by Theorem 2.1. The example is as follows: minimize

$$
J=\int_{t_{0}}^{t_{f}}\left(x_{1}-\frac{1}{2}\right)^{2} d t
$$

subject to

$$
\begin{aligned}
& \dot{x}_{1}=x_{2} u, \quad x_{1}\left(t_{0}\right)=\zeta_{1} \neq \frac{1}{2}, \\
& \dot{x}_{2}=u-x_{1}, \quad x_{2}\left(t_{0}\right)=\zeta_{2} \neq 0, \\
& |u| \leq 1, \quad t_{f} \text { fixed. }
\end{aligned}
$$

Lewis claims that the optimal solution from any initial point $\left(\zeta_{1}, \zeta_{2}\right) \neq\left(\frac{1}{2}, 0\right)$ comprises a nonsingular arc from $\left(\zeta_{1}, \zeta_{2}\right)$ reaching $\left(\frac{1}{2}, 0\right)$ at time $t_{c}$, followed by the singular arc

$$
x_{1}^{*}(t)=\frac{1}{2}, \quad x_{2}^{*}(t)=0, \quad u^{*}(t)=\frac{1}{2}, \quad \lambda_{1}(t)=\lambda_{2}(t)=0 .
$$


Clearly, $u^{*}(t)=\frac{1}{2}$ is an optimal control if the point

$$
x_{1}=\frac{1}{2}, \quad x_{2}=0
$$

is on the optimal trajectory, since the positive semidefinite cost function along this subare is zero. However, Lewis neglected to observe that $u^{*}=0$ is also an optimal control for this case.

A thorough study of this problem shows that $u^{*}=0$ is a more robust singular subarc in this problem. Furthermore, there exists a large class of initial conditions for which $u^{*}=0$ is the only optimal singular control and, except for one point in the singular state space, Theorem 2.1 is applicable. Thus, this example does not diminish the applicability of Theorem 2.1. However, this is a very useful example for studying the interrelationship of problem and arc orders. Application of the definitions and procedures of Section 2 imply that the problem is first order, i.e.,

$$
\begin{aligned}
& H=\left(x_{1}-\frac{1}{2}\right)^{2}+\lambda_{1} x_{2} u+\lambda_{2}\left(u-x_{1}\right), \\
& \dot{\lambda}_{1}=-H_{x_{1}}=-2\left(x_{1}-\frac{1}{2}\right)+\lambda_{2}, \quad \dot{\lambda}_{2}=-\lambda_{1} u, \\
& H_{u}=\lambda_{1} x_{2}+\lambda_{2}, \quad \dot{H}_{u}=\lambda_{2} x_{2}-2 x_{2}\left(x_{1}-\frac{1}{2}\right)-\lambda_{1} x_{1}, \\
& \ddot{H}_{u}=-2 x_{1} \lambda_{2}+4 x_{1}\left(x_{1}-\frac{1}{2}\right)+\left[\lambda_{2}-2\left(x_{1}-\frac{1}{2}\right)-2 x_{2}^{2}-2 \lambda_{1} x_{2}\right] u,
\end{aligned}
$$

or

$$
\ddot{H}_{u} \equiv A(t, x, \lambda)+B(t, x, \lambda) u
$$

implies that $u$ first appears explicitly in $\ddot{H}_{u}$ (implying that problem order is equal to one). The Lewis singular arc of Eq. (13) is of the second order, i.e.,

$$
\begin{aligned}
\ddot{H}_{u}\left[t, x^{*}(t), \lambda^{*}(t), u^{*}(t)\right] \equiv & 0, \quad \text { on }\left(t_{1}, t_{f}\right], \\
\ddot{H}_{u}\left[t, x^{*}(t), \lambda^{*}(t), u^{*}(t)\right]= & u^{* 2}\left(-3 \lambda_{1}-6 x_{2}\right)^{*} \\
& +u^{*}\left[4 x_{1} \lambda_{1}-4 \lambda_{2} x_{2}+8 x_{2}\left(x_{1}-\frac{1}{2}\right)\right. \\
& \left.+8 x_{1} x_{2}\right]^{*},
\end{aligned}
$$

or

$$
\begin{aligned}
& \ddot{H}_{u}=0 \cdot u^{* 2}+0 \cdot u^{*} \Rightarrow\left[(\partial / \partial u) H_{u}^{(3)}\right]^{*}=0, \\
& H_{u}^{(4)}\left[t, x^{*}(t), \lambda^{*}(t)_{s} u^{*}(t)\right]=-6 u^{* 3} \\
& +u^{* 2}\left[-5 \lambda_{2}+6\left(x_{1}-\frac{1}{2}\right)+14 x_{1}+8 x_{2}^{2}+4 \lambda_{1} x_{2}\right]^{*} \\
& +u^{*}\left[-8 x_{1}^{2}+4 x_{1} \lambda_{2}-4 x_{1}\left(x_{1}-\frac{1}{2}\right)\right]^{*}
\end{aligned}
$$

or

$$
H_{u}^{(4)}=-6 u^{* 3}+7 u^{* 2}-2 u^{*}
$$


which implies that

$$
\left[(\partial / \partial u) H_{u}^{(4)}\right]^{*}=-18 u^{* 2}+14 u^{* 2}-2=0.5>0 .
$$

In Ref. 8, it was not observed that there exists another singular control for this problem:

$$
\begin{aligned}
u^{* *}(t) & =0, \quad x_{1}^{* *}(t)=0.5, \quad x_{2}^{* *}(t)=x_{2}^{* *}\left(t_{1}\right)-0.5\left(t-t_{1}\right), \\
\lambda_{1}^{* *}(t) & =\lambda_{2}^{* *}(t)=0 .
\end{aligned}
$$

In fact, this singular control also allows the state of the singular arc defined in Eq. (13) with no increase in the cost function, and thus is a more robust singular arc. This becomes clear when one considers a phase plane analysis for this example. There exist four obvious optimal controls: $u=+1$ (nonsingular), $u=-1$ (nonsingular), $u=0$ (first-order singular arc), and $u=\frac{1}{2}$ (second-order singular arc). The state trajectories for the nonsingular arcs emanating from $\left(\zeta_{1}, \zeta_{2}\right)$, with $t_{0}=0$, are

$$
\begin{aligned}
& x_{1}=\left(\zeta_{1}-1\right) \cos t+\zeta_{2} \sin t+1, \quad u=+1, \\
& x_{2}=-\left(\zeta_{1}-1\right) \sin t+\zeta_{2} \cos t, \quad u=+1, \\
& x_{1}=\frac{1}{2}\left(\zeta_{1}-\zeta_{2}+1\right) e^{t}+\frac{1}{2}\left(\zeta_{1}+\zeta_{2}+1\right) e^{-t}-1, \quad u=-1, \\
& x_{2}=-\frac{1}{2}\left(\zeta_{1}-\zeta_{2}+1\right) e^{t}+\frac{1}{2}\left(\zeta_{1}+\zeta_{2}+1\right) e^{-t}, \quad u=-1 .
\end{aligned}
$$

The following relations hold in the phase plane

$$
\begin{array}{rr}
\left(x_{2}-1\right)^{2}+x_{2}^{2}=\left(\zeta_{1}-1\right)^{2}+\zeta_{2}^{2}, & u=+1, \\
\frac{1}{2} x_{2}^{2}=\frac{1}{2} x_{1}^{2}+x_{1}+\left(\frac{1}{2} \zeta_{2}^{2}-\frac{1}{2} \zeta_{1}^{2}-\zeta_{1}\right), & u=-1 .
\end{array}
$$

The resultant phase plane is shown in Fig. 1.

This example illustrates an interesting phenomenon that gives insight into the order of a singular arc and singular control in general. In the phase plane of Fig. 1, nonsingular subarcs are optimal for the following set:

$$
\left(x_{1}, x_{2}\right) \in S_{1} \equiv\left\{\left(x_{1}, x_{2}\right) \mid x_{1} \neq 0.5\right\} \text {, }
$$

which is all of $R^{2}$, except the line $x_{1}=0.5$. The first-order singular subarc $u^{*}=0$ is optimal for the set

$$
\left(x_{1}, x_{2}\right) \in S_{2} \equiv\left\{\left(x_{1}, x_{2}\right) \mid x_{1}=0.5\right\},
$$

while the second-order singular subarc $u^{*}=\frac{1}{2}$ is an optimal control only for the set

$$
\left(x_{1}, x_{2}\right) \in S_{3} \equiv\left\{\left(x_{1}, x_{2}\right) \mid x_{1}=0.5, x_{2}=0\right\} .
$$

Note that $S_{2}$ is a one-dimensional subset of $R^{2}$, and $S_{3}$ is a zero-dimensional subset of $R^{2}$. Thus, as the order of the singular arc increases, the dimension 


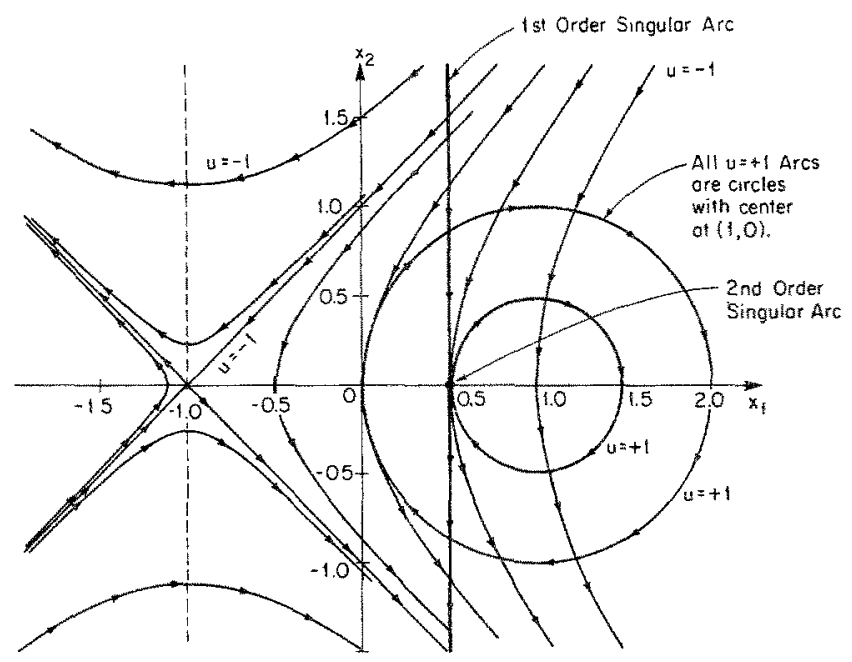

Fig. 1. Phase plane for example of Section 3.

of the space of possible states decreases. Intuitively, this is due to the algebraic constraints defined by the derivatives of the switching function in Definitions 2.1 and 2.2.

Note that, just as the first-order singular arc defines a special subspace of $R^{2}$ for this bounded control problem, the second-order singular subarc defines a special subspace of $S_{2}$. Thus, intuitively, the higher-order singular arc is a singular situation within the $q$ th order singular optimal control problem, just as the $q$ th order singular arc is a singular situation within the Oth order, nonsingular optimal control problem.

The question then arises: Is it ever necessary to employ the higherorder singular arc? In the example of Eqs. (9)-(12), the minimum cost can always be attained with a nonsingular arc (or multiple nonsingular arcs), followed by the first-order singular arc for sufficiently large $t_{f}$. However, the problem has no terminal state boundary conditions. If the following terminal condition is added to the problem:

$$
x_{2}\left(t_{f}\right)=0,
$$

then, for many initial conditions and sufficiently large $t_{f}$, the optimal control is

$$
\begin{array}{ll}
u^{*}(t)=+1 \text { or }-1, & t \in\left[t_{0}, t_{1}^{t}\right), \\
u^{*}(t)=0, & t \in\left(t_{1}^{\prime}, t_{2}^{\prime}\right), \\
u^{*}(t)=0.5, & t \in\left(t_{2}^{\prime}, t_{f}\right],
\end{array}
$$




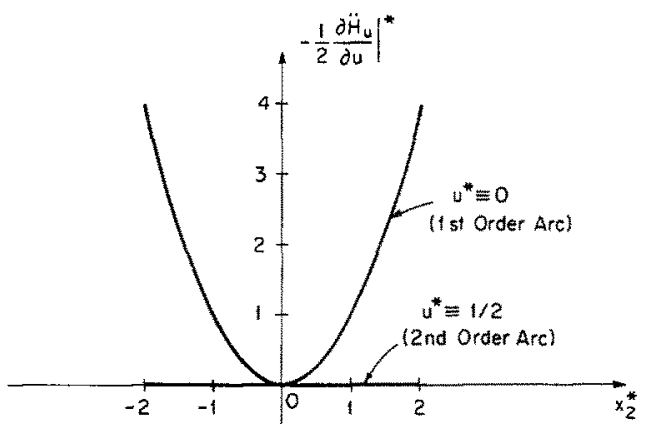

Fig. 2. Generalized Legendre-Clebsch function for example of Section 3.

i.e., a nonsingular arc/first-order singular arc/second-order singular arc sequence. Note that there may exist initial conditions which require multiple nonsingular arcs before joining the singular arc. Thus, the addition of the boundary condition (33) causes $u^{*}=\frac{1}{2}$ to be a unique singular control.

With respect to the junction condition of Theorem 2.1 , the hypotheses of the theorem are satisfied at the junction of each nonsingular arc and the singular $\operatorname{arc} u^{*}=0$, except when

$$
x_{1}\left(t_{c}\right)=\frac{1}{2} \quad \text { and } \quad x_{2}\left(t_{c}\right)=0 .
$$

It is interesting to note that this is the only point in state space which admits two singular arcs with equal cost functions, and it is the only point on the first-order singular arc where the strengthened Legendre-Clebsch condition does not hold (see Fig. 2). Thus, Theorem 2.1 is applicable to most nonsingular-singular junctions for this example, and the only point in singular state space at which it is not applicable, because

$$
B\left[t_{c}, x^{*}\left(t_{c}\right), \lambda^{*}\left(t_{c}\right)\right]=0,
$$

is a very special point in the problem. In this latter sense, the lack of the applicability of the theorem for particular conditions indicates to an investigator that further analysis of such conditions is required.

\section{Singular Linear-Quadratic Problem Considerations}

The example of the previous section indicates that a deeper engineering understanding of singular problem order must be developed. Thus, it is of interest to develop a complete theory of singular order for time-invariant, singular linear-quadratic problems (SLQP's), the most basic class of singular control problems. In this section, some properties of order associated with 
SLQP will be presented without proof, because of space limitations (see Ref. 10 for the proofs and other properties of the SLQP associated with computation and functional analytic interpretations).

Consider the following problem: minimize

$$
J=\frac{1}{2} \int_{0}^{t_{f}} x^{T} P x d t
$$

subject to

$$
\dot{x}=A x+b u, \quad x(0)=x_{0} .
$$

where $(A, b)$ is a controllable pair, $P$ is symmetric, $u$ is scalar, $P, A, b$ are constant matrices, and $x$ is an $n$-vector. The controllability assumption is imposed so that the problem is a reasonable control problem; e.g., if $(A, b)$ is not a controllable pair, then the cost function could be completely independent of the control action and independent of the path, since $u$ does not appear explicitly in Eq. (35).

It is straightforward to show that the order of the problem and the order of the arc are always the same for the class of problems defined in Eqs. (35) and (36). Since the order of the problem can be determined a priori, all order-dependent results for the SLQP can be determined a priori, which is not the case for more general classes of problems. Thus, order-dependent results for the SLQP are strictly a function of interrelationships between $P$, $A, b$ of Eqs. (35) and (36).

In linear-quadratic controller design, the matrix $P$ is usually assumed to be positive semidefinite (at least). In this case, it can be shown that, if a singular optimal control exists, then the order of the singular problem is less than or equal to the dimension of the state space, i.e., $q \leq n$. Thus, if $P \geq 0$, one is guaranteed that the process of differentiation of the switching function will terminate in at most $2 n$ steps. Actually, by employing properties of linear systems, one can develop a computer determination of order for this class of problems, as shown in Ref. 10.

If $P$ is not a positive semidefinite matrix, then it can be shown that the order of the problem is either less than or equal to $n$ or infinity, i.e., $q \leq n$ or $q=\infty$. Furthermore, an $\infty$-order SLQP can be characterized completely as follows.

Theorem 4.1. The optimal control for the SLQP of Eqs. (35) and (36) is $\infty$-order iff there exists a symmetric matrix $F$ which satisfies

$$
\begin{aligned}
P & =A^{\mathrm{T}} F+F A, \\
F b & =0 .
\end{aligned}
$$


The proof of Theorem 4.1 shows that the conditions (37) and (38) are associated with the representation of the integral in Eq. (35) as an exact differential form. Thus, an $\infty$-order SLQP (i.e., the control never appears explicitly in a derivative of the switching function) occurs only if the problem defined by Eqs. (35) and (36) is a disguised Mayer problem [for, if Eq. (35) is an exact differential, then it can be expressed as a function of $x_{0}, t_{f}, x\left(t_{f}\right)$ only]. In such cases, there exists an infinity of controls which will produce the same cost [since the system is controllable and only a function of $t_{f}$ and $x\left(t_{f}\right)$ must be minimized]. Finally, it can be shown that there exists a unique minimum-norm optimal control for the $\infty$-order SLQP. However, this control cannot be determined by differentiating the switching function (since $u$ never appears explicitly). Methods for determining the minimum-norm, $\infty$-order singular control are presented in Ref. 10.

Infinite-order singular optimal controls for SLQP's occur when the optimal control for the original problem is nonunique. Recall from Section 3 that nonuniqueness problems were also associated with the order of the problem and the arcs. Intuitively, these properties indicate that there exists a strong connection between singular problem and arc order and uniqueness of optimal controls.

\section{Concluding Remarks}

Singular optimal control problem order properties have been presented for linear-quadratic problems and a bilinear example. These results indicate that the order and the genericity of the order are valuable engineering concepts in the analysis of singular optimal control problems, contrary to the conclusions presented in Ref. 8. Further work is required in the area of singular control order, especially the development of necessary conditions for junctions of optimal subarcs at points where the generalized LegendreClebsch condition is satisfied trivially (i.e., with equality, as opposed to strict inequality).

\section{References}

1. KELLEY, H. J., A Second Variation Test for Singular Extremals, AIAA Journal Vol. 2, No. 8, 1964.

2. KOPP, R. E., and MOYER, H. G., Necessary Conditions for Singular Extremals, AlAA Journal, Vol. 3, No. 8, 1965.

3. KEllEY, H. J., KOPP, R. E., and MOYER, H. G., Singular Extremals, Topics In Optimization, Edited by G. Leitmann, Academic Press, New York, New York, 1967. 
4. RobBins, H. M., A Generalized Legendre-Clebsch Condition for the Singular Cases of Optimal Control, IBM Journal, Vol. 11, pp. 361-372, 1967.

5. MCDANelL, J. P., and Powers, W. F., Necessary Conditions for Joining Optimal Singular and Nonsingular Subarcs, SIAM Journal on Control, Vol. 9, No. 2, 1971.

6. KRENER, A. J., The High-Order Maximum Principle and Its Application to Singular Extremals, SIAM Journal on Control, Vol. 15, pp. 256-293, 1977.

7. REBHUHN, D., On the Stability of the Existence of Singular Controls under Perturbation of the Control System, SIAM Journal on Control, Vol. 16, pp. 463-472, 1978.

8. LEWIS, R. M., Definitions of Order and Junction Conditions in Singular Optimal Control Problems, SIAM Journal on Control, Vol. 18, No. 1, 1979.

9. Bell, D. J., and JACOBSON, D. H., Singular Optimal Control Problems, Academic Press, New York, New York, 1975.

10. Bortins, R., Boustany, N. M., and POWERs, W. F., The Infinite-Order Singular Problem, University of Michigan, CICE Report No. 80-1, 1980. 